\title{
Flocculation of kaolin particles by two typical polyelectrolytes: A comparative study on the kinetics and floc structures
}

\author{
Jianfeng $\mathrm{Yu}^{*}$, Dongsheng Wang, Xiaopeng Ge, Mingquan Yan, Min Yang \\ State Key Lab of Environmental Aquatic Chemistry, Research Center for Eco-Environmental Sciences, \\ Chinese Academy of Sciences, Box 2871, Beijing 100085, China
}

Received 22 January 2006; received in revised form 18 April 2006; accepted 24 May 2006

Available online 3 June 2006

\begin{abstract}
The flocculation kinetics of kaolin particles induced by two polyelectrolytes is studied by using small-angle laser light scattering (SALLS). Two different methods, image analysis and SALLS, are used to calculated the fractal dimensions of flocs formed under different flocculation mechanisms. For a high charge density of polydiallyldimethylammonium chloride (PDADMAC), the initially flocculation rates are slow due to the quite low molecular weight. Smaller and more compact flocs are in the particle-particle connections, and restructuring of the flocs occurs in the flocculation process. With cationic polyacrylamide C498 of very high molecular weight and low charge density, however, the initially flocculation rates are much higher due to its rapid adsorption on kaolin particles, but it will take the adsorbed polymer a much longer time to reach equilibrium due to re-conformation. High potentialities of adsorption prevent the particles from entering the interior of the floc structure or rearrangement, which results in a more open floc structure. Different underlying flocculation mechanisms are evident for these two kinds of polyelectrolytes, in which charge neutralization is mainly involved for the low molecular weight and high charge density polymer of PDADMAC while polymer bridging is suggested to be the dominant mechanism for the high molecular weight polyelectrolyte of C498.
\end{abstract}

(C) 2006 Elsevier B.V. All rights reserved.

Keywords: Polyelectrolyte; Kinetics; Floc structure; Charge neutralization; Bridging

\section{Introduction}

The synthetic organic polymers have been widely used in the industrial solid-liquid separation process. Practical examples include applications in water and wastewater treatment, mineral processing, paper making, etc. [1-3]. While most of flocculation practices are still depended on the experiences, considerable progress has been made in the understanding of the particle flocculation mechanisms induced by polymers recently [4-7]. The polymers can destabilize the colloidal particles through charge neutralization, electrostatic patch, bridging, or depletion flocculation. The electrostatic patch was first introduced by Gregory [8] to explain the coagulation of low charge density particles with oppositely charged polymers of very high charge density. The net residual charge of the polymer patch on one particle surface can attach to the bare part of an oppositely

\footnotetext{
* Corresponding author.

E-mail address: mailyujf@hotmail.com (J. Yu).
}

charged particle. Depletion flocculation is induced by the low adsorption polymers with high concentrations, where exists the osmotic pressure between polymer-rich and particle-rich phases due to the difference of polymer concentration [9-11].

It has been accepted that the bridging mechanism is operating in the flocculation by nonionic polymers. However, the situation becomes more complex in the systems containing colloids and polyelectrolytes of opposite charges. For example, the aggregation kinetics and aggregate structures of hematite have been studied in the presence of polyacrylic acid of different molecular weights, polyacylamide and partially hydrolyzed polyacrylamide of high molecular weight [4-6]. These results show that, regardless of polyelectrolytes with low charge density or high molecular weight, the aggregation kinetics can be well explained by the charge neutralization mechanism, and there is no evidence of polymer bridging. However, Yu and Somasundaran [12] and Fan et al. [13] investigated the flocculation of alumina particles with a dual polymer combination, and found that polymer bridging contributed to the primary floc formation for the system. Furusawa et al. [14] found that 
in the low concentration polyelectrolytes, the anionic latex was flocculated by the bridging effect of cationic polyelectrolytes. In recent literature, bridging flocculation is believed to be the most common aggregation mechanism in the system of colloidal particles and opposite polymers [15-17]. Although controversies still exist, the results obtained under the given conditions can shed light on these two mechanisms in the model systems, respectively. It is therefore important to make further and more extensive studies on the difference between the polymer bridging and charge neutralization, especially for the above mentioned colloidal particles with opposite charge polymers.

In the previous research, the mechanisms of flocculation of colloidal particles were mostly based on the final results of flocculation, e.g., the residual turbidity, the settling rates and the viscosity [12-14]. However, little work has been focused on the flocculation kinetics and floc structure [18-20]. Dynamic light scattering and static light scattering have been applied to monitor the size evolution of aggregate process. For example, the aggregation kinetics of hematite and aluminum oxides was studied using dynamic light scattering commonly referred to as photo correlation spectroscopy (PSC) [4-6,21]. Recently, static light scattering, such as small-angle laser light scattering (SALLS), has also been employed to determine on-line floc size of the alum-polystyrene flocculation [22], the sludge flocculation [23-24], the salt-latex flocculation [25-26], and the coagulation of raw water with rich natural organic matter [27-28]. Furthermore, SALLS has also been extensively utilized in acquiring information on the aggregate structures in terms of the mass fractal dimension. This method has been successfully used to rapidly determine the fractal dimension of the sludge floc structure [24,29-31], the salt-particle floc structure $[25,26,32,33]$, and the polymer-alumina floc structure [9-11,15-17]. In addition, the traditional technique of image analysis was also used to determine the floc structure by fractal method [34-36].

Cationic polymers, such as polyacrylamide and polydiallyldimethylammonium chloride (PDADMAC) are widely investigated in synthetic colloidal systems and in water treatment. An in-depth study of their flocculation kinetics and floc structures in aggregation can help to a better understanding of the flocculation mechanisms. In this investigation, the flocculation kinetics of the negative kaolin particles induced by these two polyelectrolytes is compared using SALLS. Their floc structures are studied using two different methods, image analysis and the mass fractal dimension of SALLS, and the flocculation mechanisms are also discussed. The optimum dosages are determined by the residual turbidity and zeta potential measurement.

\section{Materials and methods}

\subsection{Materials}

\subsubsection{Kaolin suspension}

Kaolin clay was used as a model suspension. The stock suspension of kaolin particles was prepared in deionized water, which was similar to Yukselen and Gregory [37]. The solid content was determined gravimetrically and found to be $68.75 \mathrm{~g} / \mathrm{l}$. The test suspensions were prepared by diluting the stock suspension to a desired concentration of $100 \mathrm{mg} / \mathrm{l}$. All working suspensions contained $1 \mathrm{mM}$ of $\mathrm{NaNO}_{3}$ and $\mathrm{NaHCO}_{3}$, respectively, to provide fixed concentrations of electrolyte and alkalinity. The particle size distribution of the kaolin suspension was measured by Mastersizer 2000 (Malvern Co., UK). The particles had a $d(50)$ diameter of about $1.0 \mu \mathrm{m}$ with a relatively narrow size distribution. The zeta potential was measured by Zetasizer 2000 (Malvern Co., UK). The clay suspension was negatively charged at about $-60 \mathrm{mV}$. These results were easily reproduced in all experiments.

\subsubsection{Polyelectrolytes}

Two commercial cationic polyelectrolytes, polydiallyldimethylammonium chloride and polyacrylamide, were used in flocculation tests based on their typical molecular sizes and charge density. PDADMAC (Taicang Floc Company, China) was low molecular weight of about 1.2 million and high charge density of $100 \%$. C498 (Cytec industries Inc, USA) was a cationic copolymer of acrylamide and acryloxyethyltrimethylammonium chloride with high molecular weight of around eight million and low charge density of $55 \%$. The cationic polymers were all prepared in a concentration of $0.1 \%$ (W/W) solution according the method provided by the companies.

\subsection{Methods}

\subsubsection{Flocculation procedure}

All the flocculation experiments were conducted at room temperature. The test suspensions were contained in 1-1 beakers. Using standard jar tests, including rapid mix at $200 \mathrm{rpm}$ for $2 \mathrm{~min}$, slow stirred at $40 \mathrm{rpm}$ for $20 \mathrm{~min}$, and settling for $10 \mathrm{~min}$, the optimum amounts of polyelectrolytes were determined. During this procedure, a small sample was taken after the $1 \mathrm{~min}$ rapid mix period for the determination of zeta potential, and after flocculation samples were withdrawn from about $25 \mathrm{~mm}$ below the water level for residual turbidity measurements (Hach $2100 \mathrm{~N}$ turbidimeter, USA).

\subsubsection{Floc size measurement}

Mastersizer 2000 was used to monitor the dynamics of floc size as the flocculation proceeded. Jar tests were conducted in a similar fashion except that the slow stirring at $40 \mathrm{rpm}$ for $30 \mathrm{~min}$ was applied for a better understanding of the flocculation kinetics and the floc structures. The suspension was measured by continuous recycle of water flowing through the sample cell of the instrument. A peristaltic pump (BT00-300, Longer Precision Pump, China) with $5 \mathrm{~mm}$ internal diameter at a flow rate of $2.01 / \mathrm{h}$ was used to draw the suspension. The pump was located downstream of the instrument to prevent disturbing the flocs prior to measurement. Samples were withdrawn from the same position in the jar, where was located between the impeller and the top of suspension. The above conditions were maintained throughout all the tests, so that the results could be compared to each other. 


\subsubsection{Floc structure determination}

Details in the theory of the mass fractal dimension using SALLS have been reported in a few literatures [30,31,38], and a brief overview will here be given in order to provide a basis on our experiment. The light scattering technique involves measurement of light intensity $I$ as a function of the scatter vector $Q$. The vector is defined as the difference between the incident and scattered wave vectors of the radiation beam in the medium, which is given by [30]

$Q=\frac{4 \pi n \sin (\theta / 2)}{\lambda}$

where $n, \theta$, and $\lambda$ are the refractive index of the medium, the scattered angle, and the wavelength of radiation in vacuum, respectively.

The mass fractal dimension $D_{\mathrm{f}}$ can be determined from the negative slope of a plot of $\log$ scatter intensity as a function of $\log$ scatter vector:

$I \propto Q^{-D_{\mathrm{f}}}$

This relationship exists provided that [30]: (i) the primary particles making up the flocs is smaller than the wavelength of scatter light and can be considered to be independent scatterers; (ii) the length scale of investigation is much larger than the primary particles and much smaller than the floc aggregates; (iii) the polydispersity effects are not significant.

The two-dimensional fractal dimension $D_{2}$ was measured using the technique of image analysis. The system was consisted of digital charge coupled device camera (CCD), monitor, and image capture and analysis software. The procedures of image analysis were adapted from Chakraborti et al. [34,35] and Kim et al. [36]. The two fractal dimension can be determined by the slope of a plot of $\log$ projected area $A$ as a function of log largest length $l$ :

$A \propto l^{D_{2}}$

\section{Results and discussion}

\subsection{General flocculation behavior of polyelectrolytes}

The effects of cationic polymer concentration on the zeta potential and residual turbidity are shown in Fig. 1. For highly charged and low molecular weight polyelectrolyte, PDADMAC, the zeta potential of the kaolin particles increases significantly as this polymer is added, and the charge reversal is observed at the concentration larger than $0.15 \mathrm{mg} / \mathrm{l}$. The maximal removal of the turbidity is achieved at the optimum dosage of $0.15 \mathrm{mg} / \mathrm{l}$ while the zeta potential is close to zero. Further increasing the dosage of PDADMAC, the zeta potential and residual turbidity both increase, which means that the system is restabilized again. The flocculation induced by PDADMAC occurs at the narrow dosage ranging from 0.10 to $0.20 \mathrm{mg} / \mathrm{l}$. For polymer C498, with high molecular weight and relatively low charge density, the changes of the zeta potential and residual turbidity are similar to PDADMAC, but with a higher optimum dosage of $0.40 \mathrm{mg} / \mathrm{l}$ and much broader flocculation zone from 0.20 to $0.80 \mathrm{mg} / \mathrm{l}$.

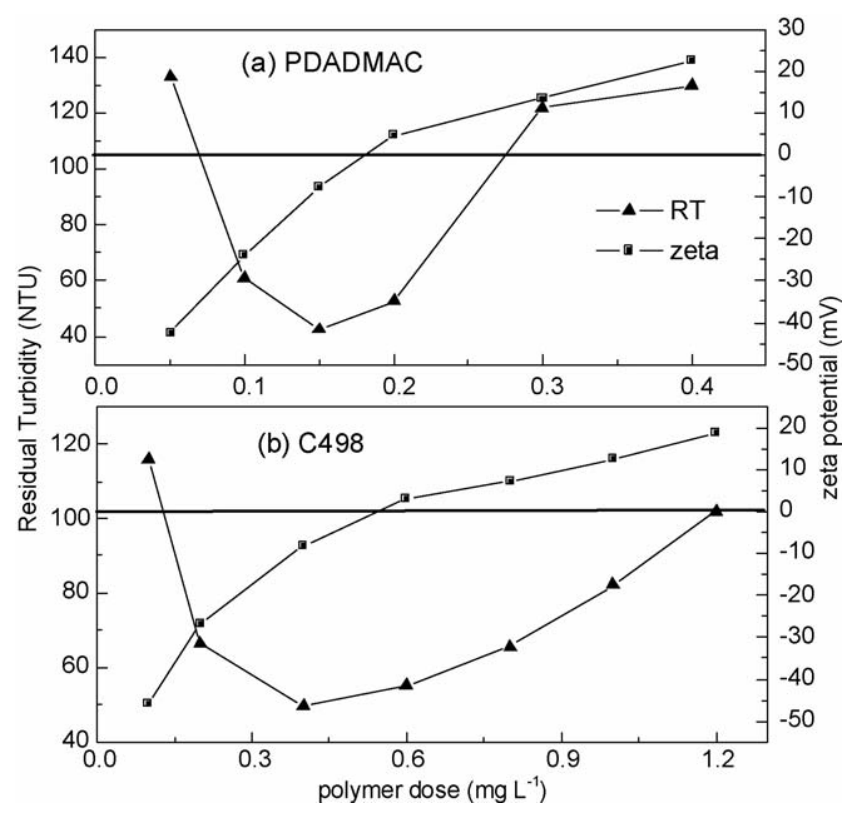

Fig. 1. Effects of the polymer dosage on the residual turbidity and the zeta potential: (a) PDADMAC; (b) C498.

It shows that the charge density and molecular weights of the cationic polymers play important roles in the coagulation of negative particles. For oppositely charged polymers and particles, two main mechanisms can be involved in the particle flocculation, i.e., charge neutralization and bridging flocculation. The optimal dosage of PDADMAC with low molecular weight is found to occur near the point of zeta potential, which is most likely attributed to simple charge neutralization. With more polymers added, the charge is reversal, implying an electrostatic repulsion between the particles. The flocculation induced by C498, however, cannot be explained simply by charge neutralization mechanism. This argument is based on the following results: (i) there is no proportional relationship between the optimal dosage of the polymers and their charge density; (ii) the flocculation zone by $\mathrm{C} 498$ is much broader than PDADMAC; (iii) flocculation kinetics and floc structures are distinguishingly different, which will be given in the later section. Hence, different mechanisms, including polymer bridging, are probably involved in the flocculation with $\mathrm{C} 498$ due to the high molecular weights.

\subsection{Effect of different mechanisms on the flocculation kinetics}

Fig. 2 demonstrates the floc size of kaolin particles changing with the time. The data presented are throughout the period of rapid mixing and flocculation. Both polymers show a typical transformation from destabilization to restabilization with the increase of polymer dosage. However, significant differences in the flocculation kinetics are evident for the two different types of polyelectrolytes.

In all the range of PDADMAC concentration, the floc size increases laggardly at the beginning of flocculation. The initial rates of the floc growth in the flocculation by $\mathrm{C} 498$, however, are 


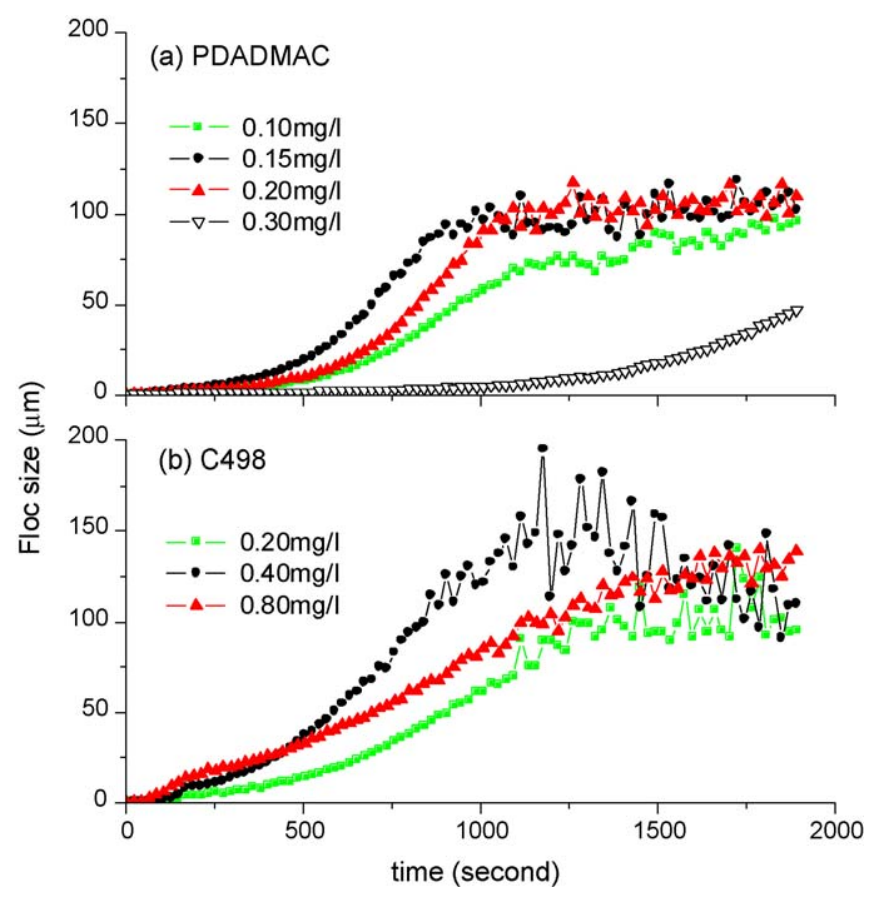

Fig. 2. Comparison of the flocculation kinetics of kaolin particles by: (a) PDADMAC; (b) C498.

greater, and no lag time is found, indicating the faster aggregate formation rates. At the optimal dosage of $0.15 \mathrm{mg} / \mathrm{l}$ PDADMAC, the floc size increases to the maximum value of about $100 \mu \mathrm{m}$ in $15 \mathrm{~min}$ and retains a steady state. Moreover, the flocculation rate of $0.15 \mathrm{mg} / \mathrm{l}$ PDADMAC is larger than that at other dosages. The floc size grows much slowly with the time at the dosage of $0.10 \mathrm{mg} / \mathrm{l}$ and even does not reach equilibrium after $30 \mathrm{~min}$ of flocculation time. Compared with PDADMAC, the changes in floc size for $\mathrm{C} 498$ are different. Besides the longer time to reach equilibrium, the final equilibrium size of $150 \mu \mathrm{m}$ at the optimal dose of $0.40 \mathrm{mg} / \mathrm{l} \mathrm{C} 498$ is also larger than that of PDADMAC. An interesting phenomenon is that the flocculation rate is increased with the dosage of $\mathrm{C} 498$ in the initial $7 \mathrm{~min}$, while the maximum rate is obtained at the optimal dose of $0.40 \mathrm{mg} / \mathrm{l}$ on the whole. This indicates that different flocculation mechanisms might be involved.

It is well known that the flocculation kinetics with polymers depends on several factors such as the mixing condition, adsorption on the particles, re-conformation of the adsorbed polymers, collision efficiency and breakage of flocs [39]. The differences on the flocculation kinetics for the two polyelectrolytes may be due to a different mechanism. No matter what mechanism is involved, the extent of adsorption and conformation is important in flocculation with polymers. As have been discussed by Gregory [19,39], the time required to adsorb a fraction of the added polymer on the surface of particles in the shearing flocculation is mainly depended on the radii of the particle and the polymer. In this experiment, the effect of particle size and the shear rate are unchanged. The difference in the adsorption time between PDADMAC and C498 may come from their different molecular sizes and the fraction of added polymers adsorbed on the particles.
The initially slow rates of aggregation with PDADMAC may be explained by its low molecular weight and a large fraction of the added polymer to neutralize the kaolin surface charge [17]. Highly charged PDADMAC, tends to adsorb in a flat state. Once adsorbed, the low molecular weight polyelectrolyte has little segments and short chains, and hence the time required to conform on the particle surface is very short and the flocculation equilibrium reaches in a relatively short time. Thus, there is less probability for bridging between particles. On the other hand, the collision radii of the kaolin particles with the adsorbed PDADMAC polymer are almost unchangeable, and connections between the destabilized particles due to charge neutralization are in direct contact, leading to a slow flocculation rate by PDADMAC.

As shown in Fig. 2(b), the floc size grows immediately as soon as the $\mathrm{C} 498$ polymer is introduced into the kaolin suspension. Unlike in the kaolin-PDADMAC flocculation system, the C498 polymer takes a much longer time to reach its maximum floc size. This can be interpreted based on the polymer bridging mechanism, which is involved with the adsorbing polymer in a transient state and in the nonequilibrium flocculation [2]. Initially, due to the adsorption of $\mathrm{C} 498$, the collision radii of particles increase and the polymer chains are in extended conformation, which may enhance the flocculation rate via bridging. It is therefore reasonable to consider the conformational change of the adsorbed polymers determining the flocculation rate $[2,12,40]$. The adsorbing polymers may change their shapes from a coiled conformation in solution to a flattened one on the surface, resulting in a longer time to reach the equilibrium.

Furthermore, the images of kaolin flocs induced by PDADMAC and C498, as shown in Fig. 3, clearly indicate that the flocs in the kaolin-PDADMAC system are smaller and more compact than those of C498. Thus, on the basis of the above analysis, it can be concluded that charge neutralization is mainly involved for PDADMAC while polymer bridging is suggested to be the dominant mechanisms for C498.

\subsection{Effect of different mechanisms on the floc structures}

Direct information about the floc structures can be obtained by analyzing the data from static light scattering. Fig. 4 demonstrates the $\log -\log$ plot of $I$ against $Q$ for the flocs at different times, and the floc size distribution is shown in Fig. 5. To eliminate the polydispersity effect, the mass fractal dimension $D_{\mathrm{f}}$ in Table 1 is restricted to the same range of $Q$ values, as have been reported by other researchers [24,31]. The mean values of $\mathrm{D}_{2}$ calculated from image analysis according to Eq. (3) are also included in Table 1 for comparison.

As shown in Fig. 4(a), slight changes are apparent in the scattering data of PDADMAC-kaolin flocs. Since the fractal dimension represents aggregates of all sizes [41], we here discuss the shape of scatter data in detail. At lower length scale of flocs $\left(Q>6 \times 10^{-4} \mathrm{~nm}^{-1}\right)$, the slopes of intensity versus $Q$ remain unchanged regardless of the flocculation time. A small upturn of the scatter data profile is apparent when $Q<6 \times 10^{-4} \mathrm{~nm}^{-1}$, and the profiles at different flocculation times become intersectant with the scatter intensity almost equal 

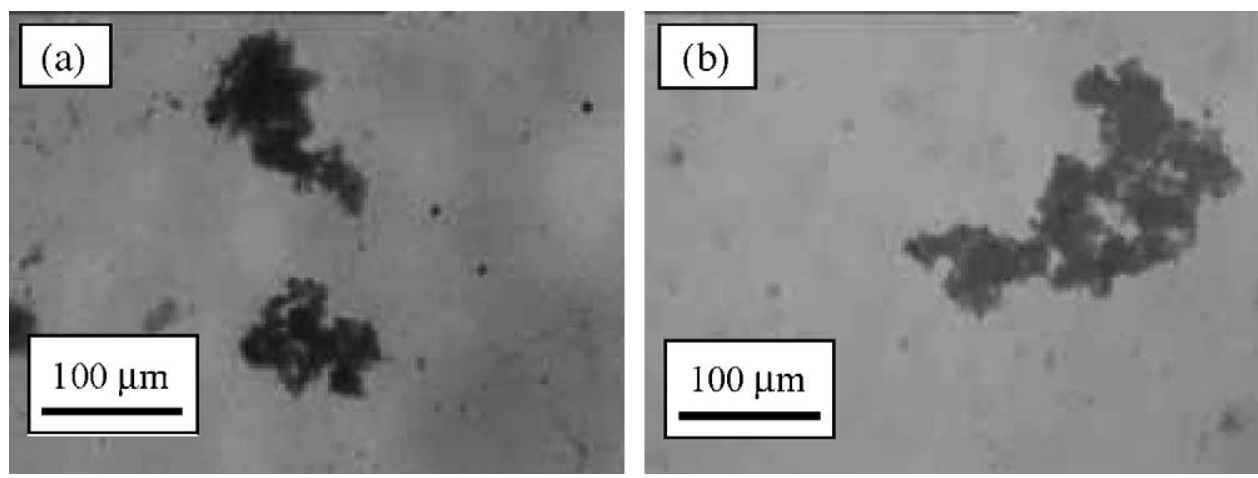

Fig. 3. Images of the flocs after the slow mixing: (a) PDADMAC, $0.15 \mathrm{mg} / \mathrm{l}$; (b) C498, $0.40 \mathrm{mg} / \mathrm{l}$.

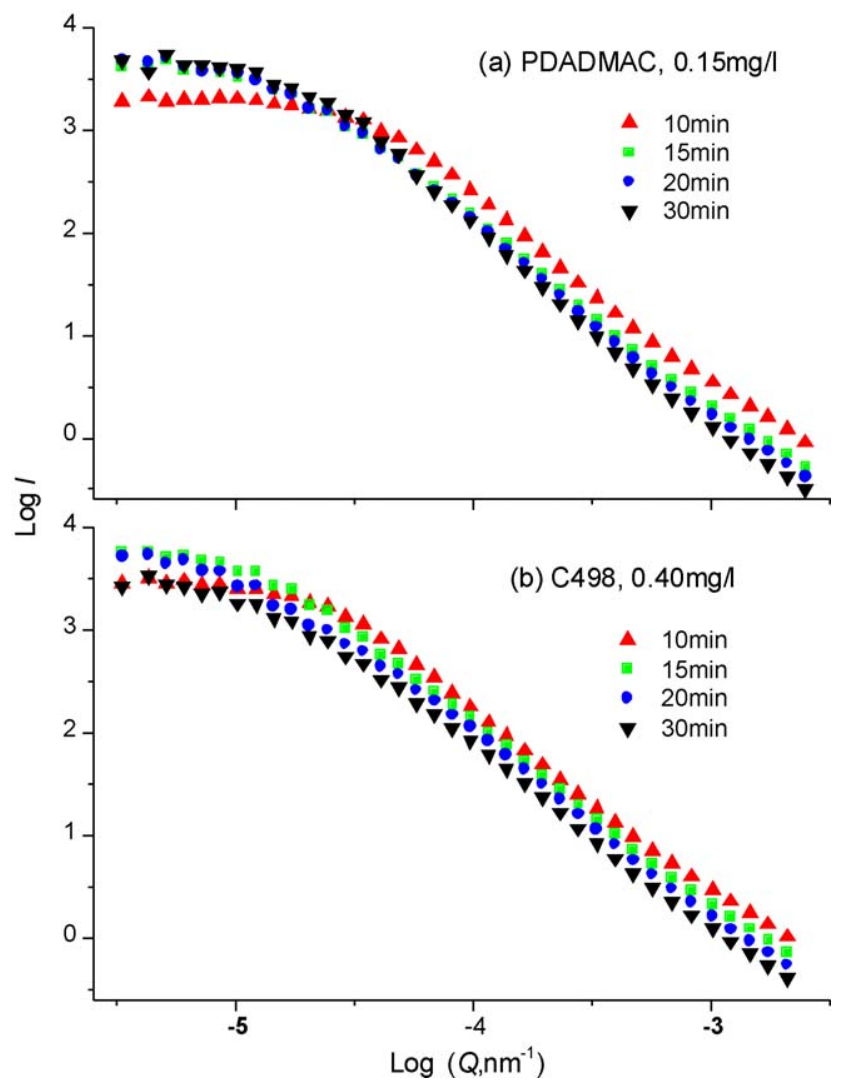

Fig. 4. The $\log -\log$ plot of $I$ as a function of $Q$ in the flocculation process at the optimal dosage: (a) PDADMAC, $0.15 \mathrm{mg} / \mathrm{l}$; (b) C498, $0.40 \mathrm{mg} / \mathrm{l}$.

Table 1

The fractal dimensions based on the small-angle light scattering and the image analysis

\begin{tabular}{llll}
\hline Polymers & Time $(\mathrm{min})$ & \multicolumn{2}{l}{ Fractal dimensions } \\
\cline { 3 - 4 } & & $D_{\mathrm{f}}$ & $D_{2}$ \\
\hline \multirow{2}{*}{ PDADMAC } & 10 & $1.83 \pm 0.01$ & \\
& 20 & $1.91 \pm 0.01$ & \\
& 30 & $2.09 \pm 0.01$ & $1.92 \pm 0.06$ \\
$\mathrm{C} 498$ & 10 & $1.79 \pm 0.01$ & \\
& 20 & $1.78 \pm 0.01$ & \\
& 30 & $1.77 \pm 0.01$ & $1.85 \pm 0.07$ \\
\hline
\end{tabular}

at about $Q=4 \times 10^{-5} \mathrm{~nm}^{-1}$. Therefore, the slope slightly increases from 1.83 at $10 \mathrm{~min}$ to 2.09 at $30 \mathrm{~min}$ in the $Q$ range of $4 \times 10^{-5} \mathrm{~nm}^{-1}$ to $10^{-3} \mathrm{~nm}^{-1}$. This phenomenon has also been observed by Selomulya et al. [26] except that the range of $Q$ in our experiment is boarder.

A drop in scatter intensity for $\mathrm{C} 498$ at the optimal dosage of $0.40 \mathrm{mg} / \mathrm{l}$ (Fig. 4(b)) is observed after the aggregate size remains stable, which can be attributed to the average mass decrease of the particles due to the sedimentation of flocs in the slow mixing. However, the reduction of the apparent volume fraction does not affect the shape of the curves. The fractal dimension $D_{\mathrm{f}}$ is maintained at $1.78 \pm 0.02$ in the $Q$ range of $4 \times 10^{-5}$ to $10^{-3} \mathrm{~nm}^{-1}$ (Fig. 4(b)), indicating a stable floc structure in the process. Calculated values of $D_{2}$ based on the image analysis are $1.92 \pm 0.06$ and $1.85 \pm 0.07$ for PDADMAC and C498, respectively, which are in accordance with the results of mass fractal dimension method.

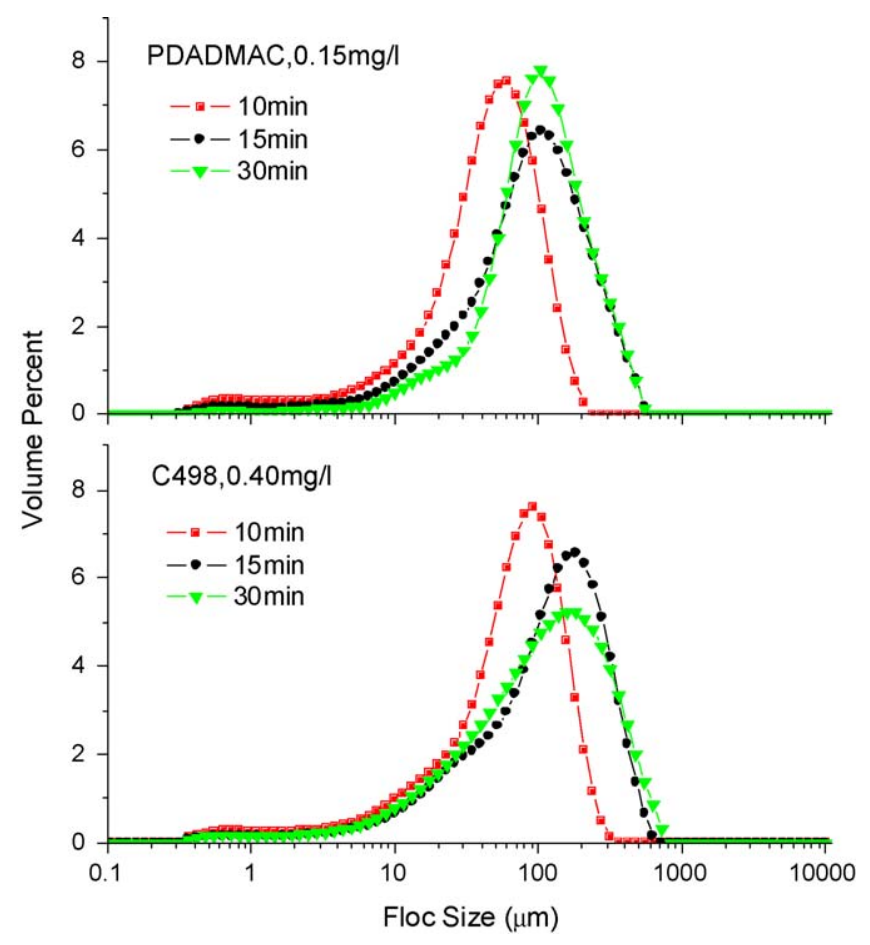

Fig. 5. The floc size distribution in the flocculation process at the optimal dosage: (a) PDADMAC, $0.15 \mathrm{mg} / \mathrm{l}$; (b) C498, $0.40 \mathrm{mg} / 1$. 
As the aggregate size increases over time, the fractal dimension usually decreases and the floc structures become more open [35]. In the flocculation with PDADMAC, however, the slope of the scatter patterns increases slightly at the intermediate scale, implying an increase in the fractal dimension. It is partly possible that the large scale flocs are joined together to form more compact and stable structures due to restructuring and rearrangement [26]. The structure of the small aggregate remains intact in the flocculation, which is consistent with the changes in floc size distribution. As shown in Fig. 5(a), the volume of the intermediate flocs slightly increases and their distribution scope becomes narrowed after $15 \mathrm{~min}$, indicating a floc structural rearrangement during the flocculation process. On the other hand, the negative kaolin particles are destabilized due to charge neutralization. A steady state size of flocs is obtained because of the balance between the weak force to form efficient collision and the breakup due to mixing-induced shear conditions. Thus, more compact flocs will be likely formed as compared with those that attach with ease.

The floc structures by high molecular weight polymer of C498 are more open in comparison with those by PDADMAC. As discussed in Section 3.2, the flocculation rates by $\mathrm{C} 498$ polymer are faster compared with those in PDADMAC. High probability of adsorption between kaolin particles and C498 prevents the particles from further entering into the interior of the cluster or the rearrangement, resulting in a more opened floc structure. As is indicated by Fig. 5(b), the floc size distribution no longer changes except that the volume fraction of the peak size is decreased at $30 \mathrm{~min}$, also indicating that a steady state has been attained after $15 \mathrm{~min}$. Fractal dimensions at the lower end of the $Q$ range $\left(Q<4 \times 10^{-5} \mathrm{~nm}^{-1}\right)$ are associated with larger assemblages, which are believed to form via diffusion limited cluster-cluster aggregation [30]. The invariable value $D_{\mathrm{f}}$ indicates diffusion limited process with no reconstruction and no rearrangement, which has been proved by Biggs et al. [16] in bridging flocculation with latex particles.

\section{Conclusions}

For the flocculation of kaolin particles by polyelectrolytes, different mechanisms can be involved based on the polymer characteristics. With the low molecular weight and high charge density polymer of PDADMAC, the flocculation rates are slow due to the slow adsorption and the direct contact between the destabilized particles. The floc structures via charge neutralization are more compact, and the rearrangement and reconstruction of flocs occur in the flocculation process. With high molecular weight and relatively low charge density polymer of C498, however, polymer bridging is the dominant flocculation mechanism. The rapid adsorption of the polymer on the kaolin particles in a transient state results in instant high flocculation rates. The C498 polymer takes a much longer time to reach equilibrium due to undergoing re-conformation. The floc structure induced by high molecular weights is more open and retains unchangeable via bridging flocculation mechanism. These results that the mechanisms are differentiated in the flocculation through the floc growth and structure may have significance for the applications of the polymers in practice.

\section{Acknowledgement}

The authors are grateful for financial support from the National 863 program under 2002AA601120.

\section{References}

[1] E. Pefferkorn, The role of polyelectrolytes in the stabilization and destabilization of colloids, Adv. Colloid Interface Sci. 56 (1995) 33-104.

[2] Y. Adachi, Dynamic aspects of coagulation and flocculation, Adv. Colloid Interface Sci. 56 (1995) 1-31.

[3] A.V. Dobrynin, M. Rubinstein, Theory of polyelectrolytes in solutions and at surfaces, Prog. Polym. Sci. 30 (2005) 1049-1118.

[4] J. Zhang, J. Buffle, Kinetics of hematite aggregation by polyacrylic acid: importance of charge neutralization, J. Colloid Interface Sci. 174 (1995) 500-509.

[5] R. Ferretti, J. Zhang, J. Buffle, Kinetics of hematite aggregation by polyacrylic acid: effect of polymer molecular weights, Colloids Surf. A: Physicochem. Eng. Aspects 121 (1997) 203-215.

[6] J. Zhang, C. Huguenard, C. Scarnecchia, R. Menghetti, J. Buffle, Stabilization and destabilization of hematite suspensions by neutral and anionic polyacrylamide, Colloids Surf. A: Physicochem. Eng. Aspects 151 (1999) 49-63.

[7] K.K. Das, P. Somasundaran, A kinetic investigation of the flocculation of alumina with polyacrylic acid, J. Colloid Interface Sci. 271 (2004) 102109.

[8] J. Gregory, Rates of flocculation of latex particles by cationic polymers, J. Colloid Interface Sci. 42 (1973) 448-456.

[9] J.L. Burns, Y.D. Yan, G.J. Jameson, S. Biggs, Relationship between interaction forces and the structural compactness of depletion flocculated colloids, Colloids Surf. A: Physicochem. Eng. Aspects 162 (2000) 265277

[10] Y.D. Yan, J.L. Burns, G.J. Jameson, S. Biggs, The structure and strength of depletion force induced particle aggregates, Chem. Eng. J. 80 (2000) 23-30.

[11] J.L. Burns, Y.D. Yan, G.J. Jameson, S. Biggs, The rheology of concentrated suspensions of depletion-flocculated latex particles, Colloids Surf. A: Physicochem. Eng. Aspects 214 (2003) 173-180.

[12] X. Yu, P. Somasundaran, Role of polymer conformation in interparticlebridging dominated flocculation, J. Colloid Interface Sci. 177 (1996) 283-287.

[13] A. Fan, N.J. Turro, P. Somasundaran, A study of dual polymer flocculation, Colloids Surf. A: Physicochem. Eng. Aspects 162 (2000) 141-148.

[14] K. Furusawa, M. Ueda, T. Nashima, Bridging and depletion flocculation of synthetic lattices induced by polyelectrolytes, Colloids Surf. A: Physicochem. Eng. Aspects 153 (1999) 575-581.

[15] S.M. Glover, Y.D. Yan, G. Jameson, S. Biggs, Bridging flocculation studied by light scattering and settling, Chem. Eng. J. 80 (2000) 3-12.

[16] S. Biggs, M. Habgood, G. Jameson, Y.D. Yan, Aggregate structures formed via a bridging flocculation mechanism, Chem. Eng. J. 80 (2000) 13-22.

[17] Y.D. Yan, S.M. Glover, G.J. Jameson, S. Biggs, The flocculation efficiency of polydisperse polymer flocculants, Int. J. Miner. Process. 73 (2004) 161-175.

[18] J. Gregory, D.W. Nelson, Monitoring of aggregation in flowing suspensions, Colloids Surf. 18 (1986) 175-188.

[19] J. Gregory, Polymer adsorption and flocculation in sheared suspensions, Colloids Surf. 31 (1988) 231-253.

[20] D.N. Thomas, S.J. Judd, N. Fawcett, Flocculation modeling: a review, Water Res. 33 (1999) 1579-1592.

[21] T.D. Waite, J.K. Cleaver, J.K. Beattie, Aggregation kinetics and fractal structure of $\gamma$-alumina assemblages, J. Colloid Interface Sci. 241 (2001) 333-339. 
[22] P.T. Spicer, S.E. Pratsinis, J. Raper, R. Amal, G. Bushell, G. Meesters, Effect of shear schedule on particle size, density, and structure during flocculation in stirred tanks, Powder Technol. 97 (1998) 26-34.

[23] C.A. Biggs, P.A. Lant, Acitvated sludge flocculation: on-line determination of floc size and the effect of shear, Water Res. 34 (2000) 2542 2550.

[24] R.M. Wu, D.J. Lee, T.D. Waite, J. Guan, Multilevel structure of sludge flocs, J. Colloid Interface Sci. 252 (2002) 383-392.

[25] G. Bushell, R. Amal, Measurement of fractal aggregates of polydisperse particles using small-angle light scattering, J. Colloid Interface Sci. 221 (2000) 186-194.

[26] C. Selomulya, R. Amal, G. Bushell, T.D. Waite, Evidence of shear rate dependence on restructuring and breakup of latex aggregates, J. Colloid Interface Sci. 236 (2001) 66-77.

[27] P. Jarvis, B. Jefferson, S.A. Parson, Breakage, regrowth, and fractal nature of natural organic matter flocs, Environ. Sci. Technol. 39 (2005) 2307-2314.

[28] P. Jarvis, B. Jefferson, S.A. Parson, How the natural organic matter to coagulant ratio impacts on floc structural properties, Environ. Sci. Technol. 39 (2005) 8919-8924.

[29] C.P. Chu, D.J. Lee, X.F. Peng, Structure of conditioned sludge flocs, Water Res. 38 (2004) 2125-2134.

[30] J. Guan, T.D. Waite, R. Amal, Rapid structure characterization of bacterial aggregates, Environ. Sci. Technol. 32 (1998) 3735-3742.

[31] T.D. Waite, Measurement and implications of floc structure in water and wastewater treatment, Colloids Surf. A: Physicochem. Eng. Aspects 151 (1999) 27-41.
[32] C. Selomulya, G. Bushell, R. Amal, T.D. Waite, Aggregation mechanisms of lates of different particle sizes in a controlled shear environment, Langmuir 18 (2002) 1974-1984.

[33] C. Selomulya, G. Bushell, R. Amal, T.D. Waite, Aggregate properties in relation to aggregation conditions under various applied shear environments, Int. J. Miner. Process. 73 (2004) 295-307.

[34] R.K. Chakraborti, J.F. Atkinson, J.E. Van Benschoten, Characterization of alum floc by image processing, Environ. Sci. Technol. 34 (2000) 3969-3976.

[35] R.K. Chakraborti, K.H. Gardner, J.F. Atkinson, J.E. Van Benschoten, Changes in fractal dimension during aggregation, Water Res. 37 (2003) 873-883.

[36] S.H. Kim, B.H. Moon, H.I. Lee, Effects of $\mathrm{pH}$ and dosage on pollutant removal and floc structure during coagulation, Microchem. J. 68 (2001) 197-203.

[37] M.A. Yukselen, J. Gregory, The reversibility of floc breakage, Int. J. Miner. Process. 73 (2004) 251-259.

[38] G. Bushell, Y.D. Yan, D. Woodfield, J. Paper, R. Amal, On techniques for the measurement of the mass fractal dimension of aggregates, Adv. Colloid Interface Sci. 95 (2002) 1-50.

[39] J. Gregory, Partilces in Water: Properties and Processes, CRC press, Taylor \& Francis Group, London, 2006.

[40] X. Yu, P. Somasundaran, Kinetics of polymer conformational changes and its role in flocculation, J. Colloid Interface Sci. 178 (1996) 770-774.

[41] A. Thill, S. Moustier, J. Aziz, M.R. Wiesner, J.Y. Bottero, Flocs restructuring during aggregation: experimental evidence and numerical simulation, J. Colloid Interface Sci. 243 (2001) 171-182. 